\title{
Comunidades virtuales y ciber-tribus: Las esferas pública y privada en el escenario reticular
}

Martínez, Gildardo*

\section{Resumen}

El objetivo de este artículo es valorar las deficiencias y virtudes de la hibridación social que producen las Nuevas Tecnologías de Información y Comunicación, considerando sus efectos sobre la escena pública y privada. Se concluye que las nuevas formas de vida pública y privada son exponentes del nuevo sentido que adquieren las formas de sociabilidad contemporánea en este fin de milenio, y la necesidad de reubicar y revisitar la relación tecnología-sociedad.

Palabras clave: Comunidad virtual, nuevas tribus, esfera pública, esfera privada, nuevas tecnología de información y comunicación.

- Director de la Escuela de Sociología de LUZ - Investigador del Centro de Estudios Antropológicos y Sociológicos de LUZ. Doctorando en Ciencias Sociales (Convenio UCV-LUZ). 


\title{
Virtual Communities and Ciber Tribes: The Public and Private Spheres of the Reticular Scene
}

\begin{abstract}
The objective of this article is to evaluate the deficiencies and virtues of the social hybridization that is produced by New Informational and Communicational Technologies, considering their effect in the public and private scenario. The conclusion is that new forms of public and private life are exponents of a new rationale manifested in the forms of contemporary socialization during this end of the millennium, and there is a need to revisit and re-assess the technology-society relationship.
\end{abstract}

Key words: Virtual community, new tribes, public sphere, private sphere, new informational and communicational technology.

\section{Overtura}

\section{Escena 1: Santa Mónica, California}

Como casi todas las noches, la fría brisa proveniente del Océano Atlántico no permitió a José y su familia dormir del todo. No porque haya olvidado cerrar las ventanas o no haya encendido la calefacción de su hogar No es un simple capricho lo que está detrás de la decisión de José. Por otro lado, para José y muchos otros habitantes de Santa Mónica, la seducción del discurso del ciberespacio es irrelevante y hasta fútil, pues la computadora $y$ todo lo que tiene que ver con ella es inexistente,... al igual que su lar. La preocupación de José y muchos otros que viven como él, no es la trascendencia del cuerpo y los sentidos en el espacio reticular; su más elemental ansiedad es la supervivencia y la subsistencia del cuerpo, de su cuerpo.

\section{Escena 2: Boston, Massachussets}

Estamos ahora en las antipodas. Sean y algunos de sus amigos de su comunidad crearon una conferencia virtual sobre los desamparados de su ciudad. Esto no pudo ser más extraordina rio cuando en menos de una semana se generaron cerca de 20.000 mensajes provenientes de residentes de la ciudad, e incluyendo personas desamparadas; los conferencistas accedieron desde sus hogares, oficinas y bibliotecas públicas. Lo significativo no estuvo sólo en la discusión en línea de esta problemática, sino en la generación de un fondo para un proyecto de hogares provisionales, The Ready-Made Home. En este caso, Sean no dudó que la comunicación mediada por computadoras ayuda a quienes en oportunidades las pueden considerar más que virtuales, inexistentes. 


\section{Escena 3: Estocolmo, Suecia}

Katty Libby o Virtual Sonya para sus allegados, posee una inteligencia superior que utiliza para fines poco convencionales. Las autoridades suecas la hicieron responsable del virus informático que afectó a 1.607 sistemas, incluyendo la Bolsa de Valores de Zurich, sólo que las pruebas fueron circunstanciales. Esta adolescente de 17 años, consigue en la cibercultura la forma social que logra transformar su reducido círculo social en un ghetto electrónico de inmensas proporciones. Claramente, tal urbanización de su espacio la ha llevado a pensar que su cuerpo es una escena, superfluo en su extensión y que todo lo significativo se concentra en los sentidos. Los mensajes que despide la pantalla de su ordenador son obscenos y placenteros a la vez, como dice Baudrillard, su autor preferido, son más visibles que lo visible y completamente llenos de información y comunicación.

\section{Ideas introductorias}

La discusión en torno a los efectos de las nuevas tecnologías de la información y la comunicación (NTIC) sobre la escena pública y privada tiene variados matices. A partir de las modificaciones en las nociones de espacio, tiempo, corporeidad, velocidad, la particular organización y articulación de ambas esferas se modifica. Para algunos, se produce la derrota de la esfera pública y la coronación de la vida individual (Adaszko, 1995; Baudrillard, 1987, 1992; Virilio, 1993); para otros, las posibilidades que brindan las nuevas tecnologías en la adición y revita- lización de los individuos a la vida civil son prometedoras (Manta y Henrique, 1996; Palacios, 1996; Reich, 1997; Rheingold, 1998; Smith, 1992). Y finalmente, están quienes consideran los fenómenos acotados irrelevantes si se les presenta como la inevitable y única condición de nuestro futuro, pues a pesar de los esfuerzos por redefinir la realidad, de impulsar las fronteras de la experiencia y la existencia a límites extremos, de vencer la propia corporeidad, de institucionalizar un mundo feliz de conectividad y comunidades digitales, la naturaleza y sus estructuras y demandas constituyen todavía los contornos concretos de la realidad para la mayoría de la humanidad (Oguibe, 1996a, 1996b; Sudaram, 1996; Gomez-Peña, 1996).

Es en este contexto de desterritorialización de unos espacios o la reterritorialización de otros, que se inscribe el presente artículo con la finalidad de valorar las deficiencias y virtudes de esta suerte de hibridación social que producen estas tecnologías.

\section{El ámbito público en el ciberespacio: ¿revitalización de la vida civil en las comunidades virtuales?}

La historia ha revelado una gradual extensión de las funciones orgánicas a través del uso de herramientas mecánicas o eléctricas. En las sociedades contemporáneas este proceso más que representar un gradiente adicional de la evolución tecnológica, la semilla para el brote de una nueva realidad o un cambio de civilización (Buxo y Rey, 1996), describe el desarrollo de las sociedades y las 
relaciones implicitas en ellas en un nuevo locus. Un fenómeno en particular, alcanzando velocidad de escape planetaria, ha logrado vencer la atracción gravitacional de la esiera terrestre hasta llegar a la inmaterialidad del espacio reticular, pero orbitando en un escenario sumamente conflictivo.

La idea de comunidad en un contexto virtual, puede entenderse como las agregaciones sociales mediadas predominantemente por computadoras y redes de telecomunicaciones; dichas agregaciones presentan tal sentido de contacto social que son capaces de posibilitar la producción de un bien común (Rheingold, 1993; Smith, 1992). No obstante el nuevo terreno de congregación, en estos sistemas de comunicación (Donath, 1999:29) pueden encontrarse los elementos sociales, económicos, políticos y culturales que constituyen los distintos dominios de una comunidad (Van Vliet y Burguer, 1987 en Fernback y Thompson, 1995). ${ }^{1}$ Los individuos en las comunidades virtuales utilizan el texto para intercambiar argumentos, reunirse en discursos intelectuales, realizar comercio, compartir ayuda emocional, realizar planes, ena- morarse, encontrar amigos y perderlos, jugar, flirtear. "Las personas en comunidades virtuales hacen casi todo lo que pueden realizar en la vida real, solo que dejamos nuestros cuerpos. Usted no puede besar a nadie y nadie puede golpearlo en la nariz, pero muchas cosas suceden en el interior de estas fronteras. Para los millones de personas que han entrado en las comunidades virtuales, la riqueza y vitalidad de las culturas de los ordenadores es atrayente e incluso adictiva" (Rheingold, 1993).

Así, la interconectividad que resulta de la comunicación mediada por computadoras (CMC) genera un espacio de interacción social que traspasa la cartografía clásica y los límites corporales, haciendo posible superar ciertas barreras físicas, sociaies e incluso psicológicas y políticas $^{2}$. Se produce, a decir de Lemos (1995) una especie de territorialité simbolique, la ruptura del paradigma moderno entre cultura y técnica; las nuevas tecnologías favorecen la apropiación técnica de lo cotidiano y también la apropiación cotidiana de la técnica, de suerte que la formación de sentimientos de conexión a intereses comunes se produzcan. Ciertos

1 Sobre el particular es pertinente añadir que aun cuando muchos procesos sociales comunes pueden adaptarse al medio ambiente reticular, otros no se transfieren tal cual: "en el clberespacio, todo el mundo esta en la oscuridad". Esta frase de Aheingold (1992) nos hace entender las limitaciones, que al interior de las comunidades virtuales, podemos encontrar. Sólo podemos intercambiar palabras. De alli que hayan surgidos ciertas "etiquetas" para la conversación ciberespacial. Las llamadas "netiquetas" que permiten expresar ciertos sentimientos y emociones a través del texto.

2 Sobre el particular, Oguibe (1996b) nos habla de las ciberdiasporas, presencias reticulares establecidas por individuos y comunidades en expatriación y que demuestran una importante ruta a través de la cual los individuos físicamente excluidos pueden evadir esos campos de obstáculos. 
rituales y ritos de pasajes que marcan físicamente a las comunidades se encuentran en línea: muerte, enfermedad, terapia sexual, al igual que discusiones de política pública y de activismo feminista o ecologista ${ }^{3}$.

La particular interacción entre humanidad y tecnologia desarrolla lo que Oldenburg llama el gran buen lugar, el espacio que es importante para nosotros en la vida diaria después del hogar y el trabajo. En el tercer lugar ${ }^{4}$. los individuos se reúnen y hablan; son los lugares donde los miembros de una comunidad interactúan con otros y se dan cuenta de los intereses que tienen en común (Oldenburg, 1997). Si separamos de esta idea la noción de localidades particulares y se exploran las relaciones e interacciones que ocurren entre los individuos, nos aproximamos a una de las cualidades especiales de las comunidades en el mundo reticular: la dimensión experiencial (Bender, 1979 en Feich, 1997).
En orden a estas ideas, el espacio virtual en el que los sujetos navegan provee múltiples rutas de interzonalidad; es decir, cual megapolis de nacionalidad indeterminada, el conjunto de comunidades virtuales gesta una interzona de solidaridad mediada (Oguibe, 1996b). Parafraseando a Alexander (1994, en Cansino y Ortiz, 1997:221), el sentimiento de solidaridad hacia sus miembros o el interés que los une, trasciende los compromisos particulares, las lealtades estrechas y los intereses sectarios. Cuando los individuos se conectan en conferencias ${ }^{5}$ deliberadas, que exploran espinosos contenidos sociales, están trasladando su experiencia de vida privada hacia la arena pública. Las conversaciones ofrecidas en comunidades virtuales revelan no tanto las actitudes de los individuos hacia determinado tópico como la efectividad del tipo de participación lograda por este medio. Desde la reproducción en línea de los problemas entre India y Pakistan y en el interior de la India misma ${ }^{6}$; la peculiar si-

3 Algunas de las comunidades virtuales que desarrollan esas conferencias se encuentran: The WELL (http://www.well.com), Electric Minds (http://www.minds.com) y CommunityWare (http://www.communityware.com.br).

4 Para Oldenburg (1997), la frase "tercer lugar" deriva de considerar el hogar como el "primer" lugar en nuestras vidas y los sitios de trabajos, como el "segundo".

5 La noción de "conferencias" es peculiar en las comunidades virtuales. Estos lugares están abiertos las veinticuatro horas del día y los conferencistas pueden conocer las diferentes respuestas al tema en cuestión, el autor de la misma y la hora en que fue realizada, evaluando el desarrollo del tema a medida que otros participantes se incorporan a ella.

Sobre el tema véase Sudaram (1996). 
tuación de los desamparados (homeless) en Santa Mónica, California ${ }^{7}$, hasta los modestos ( $८$ ?) acercamientos de alcaldias venezolanas a su comunidades a través de páginas web ${ }^{8}$, resultan en significativos brotes participativos de los individuos en la producción de un bien que es común a ellos.

Estas nuevas formas de sociabilidad surgen de experiencias compartidas en el ciberespacio, en que el intercambio simbólico de valores éticos y estéticos, imágenes, informaciones, conocimientos y hasta necesidades afectivas conforman una nueva manera de satisfacer las necesidades gregarias del hombre y del estar juntos en un territorio, no ya geográfico, sino simbólico (Bermúdez y Martínez, 2000:11).

Las comunidades virtuales inauguran una nueva dinámica del surgimiento de ideas compartidas, puesto que los grupos de interés se conforman a partir de afinidades que hablan de la presencia del sentido de pertenencia que se construye a través de las redes y creando ritos y reglas propias de interacción para el ir y el venir de la interac- ción comunicativa (Bermúdez y Martínez, 2000:11).

No obstante, la suerte de participación que se produce debe mirarse con otro cariz, pues pudiera significar que la tecnología hace más democrática la democracia, y no es así. El peso de la realidad prevalece y muchas veces los objetivos politicos pueden anular e incluso conducir la aplicación de la tecnología, haciendo que el diálogo político entre ciudadanos y representantes sea un simple survey, una couch-potato democracyque refleja acuerdo o desacuerdo desde la comodidad del espacio reticular y a través de una respuesta de oprima el botón a una opción de política pública pre-seleccionada (London, 1994).

De igual modo, la misma tecnología pudiera convertirse en un instrumento de tiranía. Cual ágora electróni$\mathrm{ca}$, la interconectividad puede materializar también una visión menos utópica, de carácter panóptica. Las conexiones que traen información a nuestros hogares, pueden ser extraídas y transmitidas a otros. Esta imagen de tecnología panóptica, opaca también sus posibilida-

7 Véase Schmitz, (1997) en Jones (1997).

8 Sobre el particular podemos mencionar los siguientes "sitios" municipales virtuales Alcaldía de Caracas: http://www.alcaldia-de-caracas.gov.ve Alcaldía del Municipio Chacao: http://www.chacao.org Alcaldia del Municipio San Francisco: http://san-francisco.org Alcaldía del Municipio Autónomo Caroni: http://www.almacaroni.com.ve Alcaldía del Municipio Pedro Maria Ureña: http://www.ventrontera.com/urena Alcaldia del Municipio Piar: http://www.cafeyocoima.com.ve/municiplopiar/pagina principal.htm. 
des en un contexto de ampliación de colectividades ${ }^{9}$.

\section{La esfera privada y el nirvana digital}

Una de las principales cualidades de los artefactos eléctricos es su inherente electroplasticidad; esto es, su capacidad para construir un rango de efectos perceptibles. En el ciberespacio, la alucinación consensual que pueden experimentar diariamente los usuarios cuando se conectan a la matrix de información y comunicación, (Gibson, 1984:51) al lado de su posibilidad liberatoria de una conciencia colectiva, resulta también en una satelización de lo real alrededor de las telecomunicaciones y los sistemas de redes de computación e información. Es la encefalización electrónica que nos refiere Baudrillard, "...la tendencia irreversible a la abstracción formal de los elementos y las funciones, a su homogeneización en un único proceso, al desplazamiento de las gestualidades, los cuerpos y los es- fuerzos hacia mandos eléctricos o electrónicos" (Baudrillard, 1987:14).

Más allá de esta transición crítica producida por la revolución de las transmisiones (Virilio, 1997 en Der Derian, 1997), hallamos el reemplazo e inversión de las formas tradicionales de vida en el territorio espacial del individuo; aquellos eventos lejanos son traídos hacia la proximidad inmediata e, irónicamente, los proporcionalmente cercanos como la familia, amistades, vecinos, se convierten en espacios espureos y hostiles. (Virilio, 1993) Siguiendo a Lasch, quien resalta la naturaleza insolidaria de los vínculos modernos, pareciera que "...nuestra sociedad, ...ha convertido las amistades profundas y duraderas, las relaciones amorosas y los matrimonios estables en algo más y más difícil de alcanzar..." (Lasch, 1976:69, citado por Bejar, 1987:75). Una interpretación más dramática nos aproxima a una sociedad intima (Bejar, 1987:74) en la era del ciberespacio, en donde el ámbito emocional se sobrepone al social.

9 Aun cuando no es de interés en este artículo destacar las caracteristicas panópticas de las nuevas tecnologías de información y comunicación, es importante resaltar cómo el fenómeno de la vigilancia adquiere dimensiones nuevas y distintivas complejizando con ello el hecho tecnológico. La vigilancia en este sentido no solamente es ahora un sistema global e integrado (Lyon y Zureik, 1996:3) sino también una red orbital y celular (Bogard, 1996:75-76). Más que nunca la visión de Bentham de su "casa de inspección" (Bentham, 1787:31) se ha confirmado "polivalente en sus aplicaciones" (Foucault, 1975:205), la ahora llamada "nueva vigilancia" (new surveillance) (Bogard, 1996: 2, 9, 55, 75-76; Lyon, 1994: 9, 53-5) es "pre-panóptica" e "hiper-panóptica" (Lyon, 1998: 100-101). La primera, por cuanto ha alcanzado la "hiper velocidad de la simulación", donde la información no sólo es recolectada y reunida sino proyectada. La segunda, pues existe en una región de ambientes electrónicos "...donde el tiempo es asincrónico y la velocidad de los flujos es crucial y donde la distancia y la proximidad están confundidas en el 'clberespacio' " (Lyon, 1998:101). 
Pero, ¿Qué significa llegar hasta el límite de lo conocido, a donde jamás habíamos llegado alcanzando lo universal? ¿Será acaso que el vacío intersticial que pretendemos cubrir maximalizando todos los significados posibles es una suerte de fantasia de muerte? (Baudrillard, 1992: 155). ¿Es el ciberespacio el opio del hombre del Siglo XXI polarizado entre la mente y el cuerpo? (Dery, 1996:252)

El frenesí informático-comunicacional es una forma de desaparición del sujeto en la privacidad de su yo en los "tiempos de cada cual" (Adaszko, 1995), es la lucha por alcanzar el horizonte de los acontecimientos, el límite de la información, el confín del pensamiento, la consumación de la singularidad. Es su contracción en si mismo que lo lleva al intimismo extremo y perpetuo y al desconocimiento del polo de alteridad, como única condición para controlar y manejar las partículas innumerables de las redes. EI individuo, en un estado de autismo reticular, llega a su nirvana, a la extinción de su yo en la pretensión de extraer toda la información del continuum virtual; de estar en todos los puntos de ese espacio y que en definitiva hace que no esté en ninguno. Extraño Narciso que "...no sueña con su imagen ideal sino con una reproducción genética al infinito" (Baudrillard, 1987:35). Quizá halla descubierto algo maravilloso o una ilusión, lo posiblemente cierto es que estas tecnologías satisfa- cen de alguna manera la necesidad de contactos e intercambios de información que las barreras espaciales y sociales obstaculizan. Lo irónico es que ahora la superautopista de las terminales tecnológicas redefinen el espacio de lo social desde su propia lógica a partir de la relación entre cada uno de los mortales con la matrix (Bermúdez y Martínez, 1999).

\section{A manera de conclusión: Tecnología $y$ sociedad revisitadas}

Las bondades del ciberespacio en la generación de un escenario de discusión pública han quedado reveladas por las oportunidades que brindan las conferencias en línea al interior de las comunidades virtuales, como articuladoras y unificadoras de una nueva forma de adaptación del escenario político a las nuevas tecnologías. El otro cariz de la utilización de las nuevas tecnologías de información y comunicación, pasa por el surgimiento de nuevas tribus, sujetos híbridos o ciber-rebeldes que logran amputarse de su yo $y$ asumen un papel absolutamente central en la sociabilidad telemática.

Sin embargo, caeriamos en la ilusión si consideramos al fenómeno del ciberespacio completamente globalizado, como quiera que su naturaleza así lo signifique. Existen zonas excluidas de los beneficios de la interconectividad no precosturas: Tecnologia y Sociedad Revisitadas", en Revista Educación y Ciencias Humanas. Año VII. Número 13. Julio-Diciembre 1999. Universidad Nacional Experimental Simón Rodriguez. Decanato de Postgrado. Caracas. 
cisamente por razones de índole técnica. En estas geografias desamparadas, los requisitos para acceder al mundo reticular pasan por cruzar los bordes de otro mundo, territorios de otredad no solamente simbólico sino de cuerpos físicos con necesidades primarias, escasamente satisfechas. Para los habitantes de estas zonas, el ciberespacio no existe, y la realidad al interior de la cual la vida transcurre no tiene espacio para una minerva virtual o un nirvana digital. La desesperanza no es la necesidad del contacto electrónico ante una sociedad que no responde a sus demandas. El aislamiento y apatía pública no es un capricho voluntario, cuando la más elemental preocupación es la sabrevivencia y subsistencia del cuerpo (Oguibe, 1996a) ${ }^{11}$.

En este contexto, existe una asintonia de efectos al interior de la cibercultura, y de ella con otros espacios de la vida social $^{12}$. A la luz de este desencuentro, podemos decir que a pesar de todo, las comunidades virtuales y las nuevas tribus son formas inusitadas del existir humano; ignorarlas, al igual que sus efectos, significa abandonar la comprensión de las nuevas formas de sociabilidad contemporáneas emergentes en este fin de milenio (Palacios, 1996); equivale, a no considerarlas exponentes de la era tecnológica que está haciendo de la cibercultura un fenómeno en expansión y, como tal, sin contomos definidos (Manta y Henrique, 1996) ${ }^{13}$.

Más que consumirnos en la ciberutopía y la autoamputación de nuestros cuerpos, y gastarnos en discursos sobre los precarios accesos a estas amenidades, que claramente para millones de personas en el mundo no son prioritarias, podemos reubicarnos al interior del marco que considera la primera razón de ser de la tecnologia: servir a la causa del desarrollo humano. (Oguibe, 1996a). No obstante tal apreciación, debemos considerar también que aun cuando el desarrollo de la tecnología no tiene una fundación ontológica en la naturaleza huma-

11 La edición número 3 de la revista electrónica "Cybersociology Magazine" (Digital Third Worlds \& Barriers to Internet Access. http://www.cybersociology.com) está dedicada a tratar en detalle el problema del "Tercer Mundo Digital" (frase acuñada por Oguibe (1996a-b).

12 Sobre el particular también queremos añadir que el fenómeno de la cibercultura revela nuevas formas de exclusión no solamente por la incapacidad de acceder a su uso, sino porque imlica a decir de Adaszco (1995) un conjunto de códigos culturales que se sustentan sobre otros a un nivel más básico "...como ser cosmovisión del mundo, formas de relacionarse con la tecnología..., sin los cuales es mucho más dificil entrar al mundo del ciberespacio".

13 Por otra parte, no podemos olvidar que también existen actores locales que impulsan sus propuestas políticas y culturales basadas en la permeabilidad de la red, tal y como ha ocurrido con los moviminetos sociales y culturales que luchan por sus derechos a ser reconocidos en su diversidad cultural y con los movimientos políticos tan to democráticos como de corte autoritario (Bermúdez y Martínez, 2000:14). 
$\mathrm{na}^{14}$, su aplicación es consecuente con las relaciones sociales existentes. Valores, normas y aptitudes así como condiciones sociales e históricas ejercen un papel relevante en el diseño de la tecnologia y en la delineación y aplicación de nuevos artefactos. Concomitantemente, usos de la tecnología controversiales o no convencionales como los que hemos descrito pueden influenciar o modificar valores.

Desde esta aproximación sociotécnica, es posible eliminar la dicotómica relación entre hombres y máquinas, sociedad y tecnología y redireccionar la aproximación de la sociedad que se está visualizando en este milenio.

Es cierto que la imagen de la sociedad actual a través de los cambios producidos por las nuevas tecnologías de información y comunicación no nos aleja mucho de visiones dantescas, para algunos, y quizá para otros, pueda representar visiones de avances importantes en la tecnología que nos conduzcan a niveles elevados de ciudadanía. En cualquiera de los casos, estamos en un escenario que tal vez sea absolutamente diferente en todos los sentidos y dimensiones inimaginables. Si la tecnología está desarrollándose 10 millones de veces más rápido que la evolución biológica ${ }^{15}$ no es de sorprender que nuestros referentes teóricos no nos ayuden a clarificar por qué lo que fue en el pasado ya no será en el futuro, pareciendo que estamos entrando en órbita sin ninguna posibilidad de retornar a tierra. Igualmente, aquí no nos estamos refiriendo a la controversia entre "verdad"

y "falsedad" o "irracionalidad" o "racionalidad", sino a la existencia de diferentes racionalidades, un "mundo de racionalidad plural" (Aibar, 1996).

Tanto uno como otro panorama, nos coloca en los extremos de una situación compleja que no podemos llegar a visualizar si no nos atrevemos a crearla. Si cerramos las puertas a los avances de la tecnología pecaríamos de hipócritas, pues la historia nos reclamaria muchos sucesos que, viéndolos hoy, no debimos haber permitido. Esta vez quizá no vemos hacia atrás $y$ pretendemos olvidarnos de eso. Vestiduras rasgadas que en esta oportunidad no nos sirven para nada.

\section{Bibliografía citada}

Adaszko, Dan (1995). Redefinición de las esferas públicas y privadas a partir de la ampliación del uso de Internet. Seminario de Informática y Sociedad. Carrera de Sociología. Facultad de Ciencias Sociales. Universidad de Buenos Aires. Http:/Www.anice.net. arinfysoc.

Aibar, E. (1996). "The Evaluative Relevance of Social Studies of Technology", en Society for Philosophy \&echnology. Volumen 1. Números3-4. Primaveraverano 1996. Http:/scholarilib.vt.edw/ ejoumals/SPT/v1_n3n4html/aibar.html

Baudrillard, Jean (1992). La ilusión del fin o la huelga de los acontecimientos.

14 Sobre este tema vease Lander, Edgardo (1994).

15 Al respecto véase Arthur, W.B. (1997). 
Edit. Anagrama, Colección Argumentos. Barcelona.

Baudrillard, Jean (1987). El otro por si mismo. Edit, Anagrama. Colección Argumentos. Barcelona.

Bejar, Helena (1987). "Autonomía y Dependencia: La Tensión de la Intimidad", pp. 69-90, en Revista Española de Investigaciones Sociológicas. Número 37. Enero-Marzo 1987. Madrid (España).

Bentham, Jeremy (1787/1995). The Panopticon Writings. Editada por Miran Bozovic. Verso. Londres/NewYork.

Bermúdez, Emilia y Martínez, Gildardo (2000). Los Estudios Culturales en la Era del Ciberespacio. Trabajo Final del Seminario Doctoral Estudios Culturales e Investigación Comunicacional. Facultad de Humanidades y Educación. División de Estudios Para Graduados. Doctorado en Ciencias Humanas.

Bermúdez, Emilia y Martinez, Gildardo (1999). "Identidades Colectivas en el Ciberespacio. Interrogantes más que respuestas", pp 52-55, en Revista Comunicación. Primer trimestre 1999. Número 105. Caracas (Venezuela).

Bogard, William (1996). The Simulation of Survelliance. Hypercontrol in Telematic Societies. Cambridge. University Press.

Buxo y Rey, María Jesús (1996). "La sociedad de la información reclama un nuevo diseño cultural", en Revista Ciberespacio. 4 de Junio. Http:/negocios. com/tendencias/artic2.htm.

Cansino, Cesar y Ortiz, Sergio (1997). "Nuevos entoques sobre la sociedad civil" pp. 211-226, en Revista Metapolitica. Volumen 1, número 2.

Der Derian, James (1997). "Interview with Paul Virilio" Speed Magazine. No. 1.4.
Http:/tunisa.sdc.ucsb.edu/speed/1.4/ articles/derderian.html.

Dery, Mark (1996). Escape Velocity. Cyberculture at the end of the century. Grove Press, New York.

Donath, Judith (1999). "Identity and Deception in the Virtual Community", pp. 29-59, en Comunnities in Cyberspace. Marc A. Smith y Peter Kollock, editores. Routledge. Londres-New York, 1999.

Fernback, Jan y Thompson, Brad (1995). Virtual Communities: Abort, Retry, Failure? Http:/www.well.com.user/ hlr/texts/vccivil.html.

Foucault, Michel (1975/1995). Discipline \& Punish. The Birth of the Prison. Vintage Books. Segunda Edición. New York.

Gibson, William (1984). Neuromancer. Ace Books. New York.

Gómez-Peña, Guillermo (1996) The Virtual Barrio The Other Frontier (or the Chicano Interneta). Ponencia presentada en la Quinta Conferencia del Ciberespacio (CYBERCONF). Madrid, España. Http://www.telefonica. es/fat/gomez.html.

Lemos, André (1995). "Les Communautes Virtuelles" en Societé, Número 45. Paris. Http://www.tacom.utba.br/ pesq/ cyber/lemos/cvirtual.html.

London, Scott (1994). Electronic Democracy. A Literature Survey. Http://www. west.net/insight/london/ ed.htm.

Lyon, David (1998). "The World Wide Web of Surveillance: The internet and OffWorld Power-Flows" pp.91-106, en Information, Comunication \& Society (iCS). Volumen 1, Número 1 , Primavera 1998, USA.

Lyon, David (1994). The Electronic Eye. The Rise of Surveillance Society. University of Minnesota Press. Minneapolis. 
Lyon, David y Zureik, Elia (1996). "Surveillance, Privacy and the New Technology" pp. 1-20, en Computers, Surveillance and Privacy. David Lyon y Elia Zureik, editores. University of Minnesota Press. Minneapolis/Londres, 1996.

Manta, André y Henrique Sena, Luiz (1996). As Afinidades Virtuais: A Sociabilidade no Videopapo. http://www.facom.ufba.br/pesq/cyber/artigos.html.

Oguibe, Olu (1996a). Forsaken geographies: Cyberspace and the New World 'Other'. Ponencia presentada en la Quinta Conferencia del Ciberespacio (CYBERCONF). Madrid, España. Http:/www.telefonica.es/fat/oguibe. html.

Oguibe, Olu (1996b) "On Digital 'Third Worlds' .And interview with Christian Hoeller", en Springer Magazine. Viena Austria. Http://www.satie.arts.usf.edu/ -ooguibe/springer.htm.

Oldenburg, Ray (1997) Our Vanishing 'Third Places'. Http://policy.gmu. Edu/edge _city/older.html.

Palacios, Marcos (1996), Modems, Muds, Bauds e Ftps: Aspectos da comunicacao no final do milenio. Http://www.facom.ufba.br/pesq/cyber /palacios/modens.html.
Reich, Emily (1997). Keyboard Communities \& Desk Chair Activists: Community And Civic Involvement Of Feminist Activists on The Internet. http://www.amherst.edu/ ereich/thesis.html.

Rheingold, Howard (1998). Virtual Communities, Phony Civil Society? Http:// Www.rheingold.com/texts/techpolitix/ civil.html.

Rheingold, Howard (1993). The Virtual Community. Http://www.rheingold. com/vc/book/intro.html.

Smith, Marc (1992). Voices from the WELL: The logic of the Virtual Commons Departamento de Sociologia. UCLA Centro para el Estudio de las comunidades en línea (Center for the Study of on-line Comunnities). Http://wwwssc net.ucls.edu/soc/csoc/papers/voices.

Sudaram, Ravi (1996) Beyond the Nationalist Panopticon: The experience of Cyberpublic in India. Ponencia presentada en la Quinta Conferencia del Ciberespacio (CYBERCONF). Madrid, España. Http://www telefonica. es/fat/sudaram.html.

Virilio, Paul (1993). The Third Interval: A Central Transition http:/www.duth. gr/ mboudourNirilio1.html. 